\title{
La Democracia en Educación y los Movimientos de Renovación Pedagógica: Evaluación de Prácticas Educativas Democráticas
}

\section{Democracy in Education and the Movements of Pedagogic Renovation: Evaluation of Democratic Educational Experiences}

\author{
Rosa Ortiz de Santos * \\ Luis Torrego \\ Noelia Santamaría-Cárdaba \\ Universidad de Valladolid, España
}

\begin{abstract}
Una de las funciones principales de las escuelas consiste en preparar al alumnado para aprender a vivir y convivir como futuros ciudadanos activos y críticos en el marco social. En este proceso, ha sido muy relevante la existencia de movimientos que han servido y sirven de referencia al conjunto de ciudadanos de la sociedad. En España, los Movimientos de Renovación Pedagógica (MRP) han defendido la educación democrática como uno de los pilares esenciales de las escuelas. En este artículo analizamos el grado de democratización de experiencias educativas promovidas por estos colectivos: Red Khelidôn, Granollers en Transición / Casal del Mestres y MeSumaría. Se ha realizado una exhaustiva revisión del estado de la cuestión referente a la educación democrática, la cual ha servido para elaborar un sistema de 16 indicadores que, tras ser validado por expertos, se ha utilizado para comprobar el cumplimiento de las bases de la educación democrática en las experiencias seleccionadas. Se concluye que se está avanzando hacia lo que entenderíamos como práctica democrática, las tres experiencias educativas pueden ser democráticas, ello denota que el modelo de los MRP es posible, y nos permite transmitir a la sociedad la importancia que tienen las actuaciones y las iniciativas que estos desarrollan para alcanzar una mejora real en la educación.
\end{abstract}

Descriptores: Democracia; Educación; Escuela, Indicadores educativos; Docente.

One of the main functions of schools is to prepare students to learn to live and live together as future active and critical citizens in the social framework. In this process, the existence of movements that have served and serve as a reference for all citizens of society has been very relevant. In Spain, the Pedagogical Renewal Movements (MRP) have defended democratic education as one of the essential pillars of schools. In this paper, we analyze the degree of democratization of educational experiences promoted by these groups: Red Khelidôn, Granollers en Transición / Casal del Mestres and MeSumaría. An exhaustive review of the state of the question regarding democratic education has been carried out, which has served to develop a system of 16 indicators that, after being validated by experts, has been used to verify compliance with the foundations of education. democratic in the selected experiences. It concludes that progress what we would understand as democratic practice, the three educational experiences can be democratic, which denotes that the education model MRP is possible, and allows us to convey to society the importance that have the actions and the initiatives that these develop to achieve a real improvement in education.

Keywords: Democracy; Education; School; Educational indicators; Teachers.

*Contacto: rosa.ortiz@uva.es

ISSN: 2254-3139

www.rinace.net/riejs/

revistas.uam.es/riejs
Recibido: $\quad 18$ de octubre 2017

$1^{\text {a }}$ Evaluación: 30 de diciembre 2017

$2^{\text {a }}$ Evaluación: 3 de febrero 2018

Aceptado: 16 de marzo 2018 


\section{Introducción}

Una de las funciones principales de las escuelas consiste en preparar al alumnado para aprender a vivir y convivir como futuros ciudadanos activos y críticos en el marco social; para ello, se torna fundamental favorecer el desarrollo integral de los discentes, educándolos en y para la democracia, siendo imperante la necesidad de propiciar prácticas y experiencias democráticas en las escuelas.

En este proceso, ha sido muy relevante la existencia de movimientos que han servido y sirven de referencia al conjunto de ciudadanos de la sociedad. Entre ellos, encontramos a los Movimientos de Renovación Pedagógica (en adelante, MRP), que han constituido una de las influencias más importantes de España en lo que a renovación educativa se refiere, especialmente a partir de la época franquista. Algunos de los principios en los que se sustentan las actuaciones de los MRP son la defensa de la escuela pública, inclusiva e integradora, la gratuidad y la laicidad de la educación, la preeminencia de una escuela científica y crítica y la participación de todas las personas que conforman la comunidad educativa.

El logro de estos principios exige el compromiso y la participación de personas concienciadas e interesadas en mejorar y renovar el panorama educativo actual y, por ende, el social. Esa transformación tiene su base en el ideal de una escuela democrática principio esencial de los MRP-. Por ello es muy relevante la capacidad de valorar y programar experiencias con un carácter democrático, con el objetivo de construir escuelas democráticas o transformar las ya existentes siguiendo los fundamentos de la democracia.

\section{Fundamentación teórica}

\subsection{Movimientos de renovación pedagógica (MRP)}

Los MRP son movimientos sociales compuestos por docentes de diferentes niveles formativos que se reúnen con el objetivo de compartir experiencias y conocimientos derivados de la reflexión de su propia práctica educativa (Martínez, 2002). A juicio de Martínez (1993), aparecieron como movimiento organizado durante la dictadura franquista. Rogero (2010) y Pericacho (2015) nos permiten conocer que la base de los MRP se encuentra en el ideario de otros movimientos e instituciones anteriores, como la Escuela Nueva, la Escuela Moderna de Ferrer i Guàrdia y la Institución Libre de Enseñanza, así como en el pensamiento y las actuaciones de pedagogos y educadores de referencia entre los que destacan Célestin Freinet o Paulo Freire.

La mayoría de grupos que se fundan inicialmente surgen en los años 70, aunque existen MRP que nacen en la década de los 60; el más reconocido es la Escola de Mestres Rosa Sensat, que coordinó la primera Escuela de Verano en 1966 (Martínez, 1993). Según Pericacho (2014), tras la Guerra Civil (1936-1939) y durante la dictadura franquista, trataron de eliminar los movimientos renovadores y las iniciativas educativas que habían surgido durante la Segunda República (1931-1936). No obstante, diversos grupos continuaron reuniéndose en clandestinidad a causa de su disconformidad con el modelo educativo imperante, caracterizado por un marcado catolicismo, la separación de los discentes por sexo y/o clase social o la eliminación de la enseñanza de lenguas distintas al castellano (Navarro, 1989). Coincidiendo con el final de la dictadura, surgieron numerosos colectivos de renovación pedagógica; el punto de convergencia que los unía consistía en 
su interés por transformar la escuela, estableciendo la democracia y la igualdad de oportunidades como sus principios fundamentales.

En la actualidad, numerosos MRP continúan actuando desde diferentes lugares de España. Algunas de sus iniciativas más reconocidas son la organización de encuentros, congresos y escuelas de verano. Estas oportunidades de reflexión, de aprendizaje y de convivencia les permiten dialogar, debatir y actuar en base a sus principales ideas educativas, entre las que subrayamos la defensa de la pluralidad en los centros educativos, de los valores de la escuela pública y de la inclusión de metodologías activas en la práctica escolar, la lucha contra las injusticias socioeducativas y la atención a las necesidades formativas del profesorado. Dichas reuniones constituyen una oportunidad para compartir y proponer iniciativas o soluciones a problemas actuales, dirigiéndose, unidos, a la renovación de la educación y el sistema educativo en general y de cada escuela en particular.

\title{
1.2. La democracia en educación y los MRP
}

Uno de los pilares en los que se sustentan las iniciativas educativas de los MRP es la democracia, considerada como "una fuerza poderosa y positiva para la revitalización de las escuelas públicas” (Apple y Beane, 1997, p. 12). Según estos autores, la democracia es una forma de gobierno que tiene como base la igualdad de oportunidades y está caracterizada por la libertad de expresión, el derecho a estar informados, la capacidad crítica de análisis y de reflexión y la preeminencia del bien común.

Si pretendemos que funcione una democracia real en la sociedad, debemos procurar la existencia y el predominio de escuelas democráticas. Para lograr establecer y llevar a cabo la democratización de las escuelas eficazmente, debemos tener en cuenta elementos clave que identificamos en Domínguez (2008):

\begin{abstract}
La democratización de los centros educativos [...] consiste en configurarlos como comunidades democráticas de convivencia, de investigación y de aprendizaje, caracterizadas por el diálogo permanente entre todos los agentes, la negociación continua para solucionar problemas y resolver conflictos, el trabajo solidario y cooperativo en pequeños grupos interactivos, el debate en asambleas, la participación activa de todos los miembros de la comunidad educativa en las deliberaciones y decisiones relevantes con el horizonte de un incremento continuo del autogobierno y de la autogestión. (p. 2)
\end{abstract}

Feito y López (2008) y Feito (2009) establecen tres pilares básicos de una escuela democrática: a) La educación de calidad para todo el alumnado, desarrollando escuelas inclusivas. b) La concepción de los discentes como los máximos protagonistas del sistema educativo. c) La participación y la colaboración activa y significativa de todas las personas que conforman la comunidad escolar.

Domínguez (s. f.) destaca otro de los requisitos del proceso de construcción de escuelas democráticas: los fines que persiguen los centros escolares también deben ser democráticos, siendo necesaria la elaboración de currículos democráticos. Para ello, es importante tener en cuenta dos concepciones diferentes: la concepción hegemónica y la concepción emergente (Domínguez, 2006). La hegemónica comprende las características de la actual escuela, mientras que la emergente se refiere a las transformaciones necesarias que se han de dar en la misma para que sea democrática.

Esta transformación es compleja, pues la concepción hegemónica no abarca solo el método de enseñanza, las ideas o las prácticas educativas que nos vienen impuestas, sino que constituye el pensamiento natural y la forma de vida de cada persona de una manera más 
profunda. Este pensamiento deriva en la creencia generalizada de que estas ideas educativas son inmutables y no existe posibilidad de cambio (Torrego, 1999).

En la pretensión de construir escuelas verdaderamente democráticas se deberá promover una educación democrática desde todos los ámbitos educativos. Existen diversas acepciones de educación democrática; tal y como señalan Belavi y Murillo (2016), el modo de entender el término "democracia", determinará en gran medida el significado que otorguemos a "educación democrática". Según Bolívar (2016), "una educación democrática, en el doble sentido de educar para la democracia y en la democracia, es a la vez un fin y un medio de la educación” (p. 70); añade que la escuela pública tiene el deber de capacitar a los discentes para participar como ciudadanos activos en la sociedad, además de incidir en que "la democracia se aprende y, por tanto, hay que enseñarla" (p. 74).

Yus (2002), por su parte, afirma que "educar para la democracia exige educar democráticamente, lo que, a su vez, significa que toda la comunidad educativa $[\ldots]$ ha de vivir diariamente la democracia” (p. 15). Domínguez (s.f.), miembro activo de la Federación de MRP de Madrid, entiende la democracia como autogobierno, defendiendo que la expresión educación democrática debería ser una redundancia, señala que:

\section{la educación debe ser democrática en sí misma y debe orientarse al fortalecimiento de la democracia económica, [...] cívica y [...] política, entendida como un incremento continuo de la libertad, de la igualdad de derechos, de la justicia, de la participación $y$ del autogobierno. (p. 1)}

Una organización relevante que aborda esta cuestión a nivel europeo es la Comunidad Europea de la Educación Democrática (en adelante, EUDEC), la cual promueve la democratización de las escuelas dentro de los estados democráticos, generando un flujo de información y programas entre centros educativos de diferentes países. EUDEC establece como principio fundamental de la educación democrática la participación de los niños y de las niñas en todas aquellas dimensiones que les afectan. Defienden que han de ser "considerados seres completos con criterio, $[\ldots]$ de tener la posibilidad de dirigir sus propios procesos de aprendizaje en base a sus intereses, capacidades y habilidades específicas, de dar su opinión, de asumir responsabilidades" (párr. 5). Completan esta idea exponiendo que

la Educación Democrática se basa en el respeto a los niños y a los jóvenes. La
Educación Democrática ocurre cuando se honra y se reconoce a los niños como
individuos que participan activamente en su camino por la educación. La Educación
Democrática es una educación basada en el sentido, la relevancia, la alegría, la
comunidad, el amor, y los derechos humanos. (párr. 1) ${ }^{1}$

Belavi y Murillo (2016) analizan tres propuestas de educación democrática partiendo de las ideas de personalidades de referencia en este ámbito. Abarcan y diferencian la educación democrática fundamentada en la deliberación y en la capacidad dialógica; la educación democrática dirigida a "fomentar el compromiso político de los jóvenes y enseñarles a implicarse políticamente" (p. 27) desarrollando la solidaridad, la igualdad, la libertad y la lucha contra la injusticia social; y la educación democrática orientada a la emancipación y la autonomía.

Partiendo de estas ideas y de lo expuesto por Puig (2000) y Parareda, Serra y Tort (2015), reflejamos nuestra propia concepción de educación democrática a través del sistema de

${ }^{1}$ https://educaciondemocratica.wordpress.com/sobre-eudec/ 
indicadores que elaboramos posteriormente como instrumento de evaluación. La búsqueda en las principales bases de datos científicas nos permite afirmar que la educación democrática y las escuelas democráticas constituyen un tema de creciente interés en la investigación educativa. Introduciendo el término "democratic education" en Web of Science (en adelante, WoS) observamos que aparece en 592 títulos de artículos (37 de ellos publicados en 2016 y 34 en 2015), mientras que en Scopus se refleja en 646 (23 publicados en 2017); por su parte, si realizamos la búsqueda de "education for democracy" en WoS obtenemos 156 resultados, de los cuales 16 se han publicado en 2016, y en Scopus 750, 26 publicados en 2017.

Esta revisión ha puesto de manifiesto la vigencia de la educación democrática para autores de referencia en el ámbito internacional como Hansen y James (2016), quienes comparan la necesidad actual de hábitos democráticos con la expresada por Dewey en la segunda década del siglo Xx, o Edelstein (2011), que estudia esta necesidad en el contexto de crisis. También se destaca la relación de esta educación para desarrollar la justicia en sociedades multiculturales (Carr, Pluim y Thésée, 2017) o en áreas de desinversión económica (Checkoway, 2012). La literatura científica internacional enfatiza el aprendizaje de la ciudadanía partiendo de la vida real (Biesta y Lawy, 2006) y la relación de este aprendizaje con la esfera pública (Biesta, 2016).

Encontramos, además, en nuestro ámbito, revistas que tratan específicamente este tema. Algunos ejemplos son el número extraordinario de 2011 de la Revista de Educación del Ministerio de Educación español, titulado "Educación, valores y democracia”, o la Revista Internacional de Educación para la Justicia Social, la cual dedicó el primer número del volumen cinco "Educación democrática y para la democracia" a trabajar esta cuestión.

Los objetivos de esta aportación científica son:

- Establecer las principales características de una escuela o experiencia educativa democrática.

- Comprobar si los MRP aplican en la práctica uno de sus principios esenciales: la defensa de la educación democrática.

- Analizar y valorar el grado de democratización de experiencias educativas promovidas por MRP.

\section{Método}

La investigación que se presenta en este artículo es de carácter cualitativo y se realiza mediante una adaptación de la técnica del análisis de contenido, que, en lugar de seguir las normas convencionales, se ajusta a las necesidades derivadas de los objetivos establecidos, como se expondrá en este apartado. Es una metodología elaborada al efecto, que no ha sido utilizada en otras investigaciones y, por tanto, no tiene resultados que permitan una homologación comparativa.

La investigación se ha estructurado en tres fases diferenciadas. En primer lugar, se realizó una aproximación al concepto de educación democrática, llevando a cabo una exhaustiva revisión de autores y autoras que trabajan este tema, que tuvo como consecuencia la elaboración de una propuesta de indicadores que pueden ser útiles para valorar experiencias o prácticas de educación democrática. En segundo lugar, se comprobó si los MRP defienden la educación democrática; para ello, se revisaron los documentos de la 
página web oficial de la Confederación de MRP y los artículos científicos escritos hasta la fecha sobre los mismos. Finalmente, se seleccionaron las experiencias educativas relacionadas con los MRP y se analizaron mediante los indicadores previamente elaborados, los cuales ya habían sido validados.

Mediante la adaptación de las categorías presentadas por Piñuel (2002), elaboramos un instrumento de evaluación constituido por una batería de indicadores referidos a distintos aspectos de la educación democrática que formulan los autores de más relevancia en este tema: Apple y Beane (1997); Puig (2000), Feito y López (2008), Feito (2009) y Parareda, Serra y Tort (2015). La selección deriva de una exhaustiva revisión del estado de la cuestión, en la que se ha puesto de manifiesto que son autores de relativa actualidad, los cuales abordan -atendiendo a lo expresado por investigadores de referencia anteriores- la cuestión de qué elementos constituyen la educación democrática y permiten, además, que sus formulaciones se trasladen a indicadores concretos y observables.

La lectura de estas obras nos posibilitó extraer los principios fundamentales que deben estar presentes en la educación democrática, construyendo un sistema de indicadores que permite analizar y comprobar si experiencias o prácticas escolares son o no democráticas, en la medida en que cada uno de los principios se vea reflejado en su desarrollo. En los indicadores 1, 6, 7, 8, 9, 14 y 15 se presentan características de la educación democrática defendidas por todas las personas mencionadas, mientras que en los indicadores restantes se recogen aportaciones concretas que aparecen citadas.

Antes de someter a contraste cada una de las experiencias, validamos el instrumento mediante consulta a cuatro expertos: dos profesores universitarios, uno de ellos coordinador de un programa de doctorado y el otro profesor de la asignatura "Métodos de Investigación e Innovación en Educación”, del grado de Educación Primaria, además de a una maestra y a un profesor de Educación Secundaria, ambos con una dilatada experiencia en MRP. Tras su valoración, se realizó un compendio entre dos indicadores que desembocaron en el número 16 y se modificó el indicador 12. El sistema de indicadores resultante, y que refleja lo que los investigadores del presente artículo concebimos como educación democrática, es el siguiente:

- Se promueve una educación pública, con intereses compartidos entre los diferentes miembros de la comunidad educativa.

- Predomina el diálogo, la negociación y las relaciones sociales basadas en el afecto entre los diferentes colectivos de una escuela (Puig, 2000).

- Destaca la cooperación, la colaboración y el trabajo en equipo en todos los niveles del colegio (Puig, 2000).

- Existen actividades colaborativas como debates (argumentación), asambleas o grupos interactivos (Puig, 2000).

- Predominan valores como la solidaridad, la colaboración, la igualdad, la empatía o el respeto, buscando una mejora del clima en la escuela y el bien común, "rasgo central de la democracia” (Apple y Beane, 1997, p. 27).

- Se disminuyen las desigualdades sociales. Los grupos son heterogéneos y diversos en cuanto a la cultura, el origen, el sexo, la clase socioeconómica, las capacidades y la edad, fomentando las relaciones interpersonales, aumentando la variedad de aportaciones y haciendo más real el entorno escolar. 
- Se favorece la convivencia positiva y el respeto entre diferentes culturas.

- Se educa en una visión positiva y crítica del conflicto, cuya resolución puede desembocar en un posible cambio, diálogo, pensamiento crítico y reflexión.

- Preeminencia de la educación para la desobediencia, desarrollando aspectos como el pensamiento crítico y libre, de modo que cada niño o niña sea capaz de generar sus propias opiniones e ideas (a veces en discrepancia con la sociedad) y actuar de manera justificada tras un previo análisis y evaluación.

- Se actúa en contra de aspectos negativos como "el racismo, la injusticia, el poder centralizado, la pobreza” (Apple y Beane, 1997, p. 28), entre otras desigualdades. Se defiende la igualdad de oportunidades para todos.

- Las personas que conforman el démos escolar tienen el deber y derecho de participar de forma activa y significativa en la vida del centro, en la toma de decisiones importantes y en la elaboración de documentos base (Feito y López, 2008 y Parareda, Serra y Tort, 2015).

- Los educandos son los protagonistas del proceso de enseñanza-aprendizaje, siendo el centro y destino de toda actividad, pues la educación ha de estar a su servicio logrando la actualización y el máximo desarrollo posible de todas sus potencialidades (Feito, 2009).

- Tiene como objetivos la formación moral del alumnado, la formación cognoscitiva y profesional, además de la formación como "ciudadano del mundo crítico, libre, justo y solidario” (Domínguez, s.f., p. 3).

- Se tienen en cuenta todas las circunstancias personales, sociales y familiares de cada uno de los discentes.

- Son frecuentes las relaciones con el entorno (próximo y lejano).

- Es autónoma, tratando de autogobernarse. Tanto las legislaciones estatales y autonómicas, como los documentos realizados a nivel de centro y de aula, deben ser abiertos y flexibles, facilitando su adaptación a la diversidad de cada escuela en concreto. Esta autonomía supone "la creación de canales de diálogo y de acción cooperativa” (Puig, 2000, p. 59).

Partiendo de las características propias de la metodología cualitativa, en el proceso de selección de las experiencias se ha realizado un muestreo por juicio, guiado por la obtención de representatividad subyacente. Se definió una característica esencial que permitiese realizar una aproximación al sector del universo en el que se pretendía profundizar (Mejía, 2000) y elegir las experiencias más representativas en función de los objetivos definidos: deberían ser experiencias promovidas por miembros de MRP, estar desarrollándose en el momento del análisis y realizarse en el ámbito español. Por este motivo, no interesó la cantidad o la extensión de la muestra, sino el grado de representatividad de la misma (Serbia, 2007). Partiendo de esta premisa, las experiencias seleccionadas fueron las siguientes:

- Red Khelidôn: persigue un proceso de enseñanza-aprendizaje cooperativo, con un enfoque inclusivo y democrático de la educación y de la sociedad. De esta red forman parte centros escolares de Aragón, Cataluña, Euskadi, Galicia e Islas Baleares. 
- Educació en Transició (Granollers en Transición / Casal del Mestres): defiende que estamos ante un profundo cambio derivado de la crisis económica, social y climática. Tiene como base de pensamiento el establecimiento de una relación cercana y respetuosa con la naturaleza, la necesidad de generar un sentimiento de colaboración y respeto entre la sociedad y la escuela, de favorecer el conocimiento de uno mismo y fomentar la educación en valores y la educación para la belleza. Se desarrolla por profesorado de centros educativos no universitarios de la ciudad de Granollers (Barcelona).

- MeSumaría: sus proyectos están basados en el empleo conjunto del arte y las Tecnologías de la Información y la Comunicación (en adelante, TIC). Generalmente están dirigidos hacia los colectivos más desfavorecidos, predominando valores como la solidaridad, la igualdad, el trabajo de las emociones y de la creatividad o el cultivo del pensamiento crítico y libre. Sus proyectos se centran en Canarias y en Extremadura.

A continuación, describimos sucintamente cómo se produjo el acceso al escenario y la negociación con los participantes. Conocimos las experiencias educativas en el XXVIII Encuentro de la Confederación Estatal de MRP, celebrado en Alicante los días 5 y 6 de marzo de 2016, donde los diferentes colectivos presentaron sus proyectos. Además de la información obtenida en dicho encuentro, se analizaron otros contenidos provenientes de fuentes como sus páginas web ${ }^{2} \mathrm{y}$ documentos oficiales, los informes de los proyectos $\mathrm{y}$ fotografías. Durante el proceso de análisis, fuimos conscientes de que la información disponible no nos permitía contrastar algunos de los indicadores de la experiencia de Educación en Transición (9, 14 y 16); por este motivo, acudimos al II Encuentro de Conspiración Educativa (MRP emergente), acontecido en el edificio Vicerrector Santiago Hidalgo (Segovia) el 21 de mayo de 2016, donde se realizó una breve entrevista informal a uno de los responsables de la experiencia.

Con el objetivo de completar este ejercicio de transparencia y ética, y valorando democráticamente la colaboración con las personas implicadas directamente en las experiencias educativas (Torrego, 2014), compartimos el análisis resultante con los participantes. Estos tuvieron la oportunidad de realizar sus aportaciones y conocer los resultados de la investigación, dotándola de una mayor credibilidad, dependencia y confirmabilidad, según los criterios de calidad y rigor establecidos por Guba (1983).

\section{Resultados}

Los resultados de la investigación derivan del contraste entre los indicadores propuestos y las tres experiencias educativas. Comenzamos con el indicador que abarca la defensa de la escuela pública y la actuación en base a intereses compartidos. Red Khelidôn lo alcanza de manera parcial, dado que no promueve una educación pública en el sentido amplio del concepto, pues trabaja con centros de carácter público, privado y concertado, incluyendo colegios confesionales. Sin embargo, las personas que conforman Red Khelidôn poseen intereses comunes: lograr una escuela fundamentada bajo los principios de inclusión,

\footnotetext{
${ }^{2}$ Red Khelidôn: http://cife-ei-caac.com/khelidon/

Educación en Transición: https://granollersentransicio.wordpress.com/

MeSumaría: http://mesumaria.org/
} 
democracia y justicia, así como fomentar el aprendizaje cooperativo a través de sus proyectos.

Por su parte, Educación en Transición y MeSumaría reflejan el indicador en su totalidad. Los objetivos principales del primero de ellos son compartidos y comunes con los de toda la comunidad; organizan seminarios en los que tratan los cambios sociales, económicos y climáticos. MeSumaría defiende la educación pública con sus programas, en los que se resalta el derecho al acceso a la educación de la totalidad de la población.

El indicador relativo al establecimiento de relaciones sociales se cumple en las tres experiencias. Una de las finalidades de Red Khelidôn consiste en lograr que los profesionales pertenecientes a dicho colectivo se reúnan, dialoguen, reflexionen y compartan sus experiencias y recursos. Esto se refleja en Educación en Transición, pues una de sus ideas principales radica en la "relación de compromiso y cooperación de la escuela con la sociedad y de la sociedad con la escuela". MeSumaría alcanza el indicador por medio de sus proyectos, en los que prima la interacción y el diálogo.

A continuación, realizamos un compendio en el análisis del tercer y el cuarto indicador, los cuales incluyen la importancia de la cooperación y de las actividades colaborativas; ambos se alcanzan por las tres experiencias. En Red Khelidôn constituyen la base de sus proyectos, basándose uno de sus objetivos en la cooperación y el trabajo colaborativo. Tratan de introducir la cooperación como un ente constante en actividades cotidianas del aula y de reflexionar sobre sus consecuencias. Esto se ve reforzado al comprobar que la inclusión del aprendizaje cooperativo en una escuela o en un aula es un requisito para formar parte de la Red.

Educación en Transición, por su parte, contempla actividades colaborativas en las que prima la interacción y la argumentación como, por ejemplo, debates, seminarios o asambleas, fomentando la cooperación y el trabajo en equipo. Estas cuestiones se desarrollan, además, en MeSumaría; un ejemplo es el proyecto Redes Sociales en Familia, en el que participan personas de diferentes núcleos familiares. Por otra parte, llevan a cabo actividades cooperativas como debates argumentativos o asambleas, que les permiten reflexionar sobre aspectos como las ventajas e inconvenientes de las redes sociales, el uso que se da a las mismas o el grado de democratización de estas.

Los valores que se reflejan en el quinto indicador -solidaridad, colaboración, igualdad, empatía y respeto- se persiguen desde las tres experiencias promovidas por MRP. Red Khelídôn realiza un profundo tratamiento de estas cuestiones, como reflejan los principios que la definen: Aprender a Cooperar y Cooperar para Aprender implica tener aprehendidos estos valores. A su vez, desde Educación en Transición se persigue el bien común, actuando contra la crisis climática, energética y económica. Asimismo, una de las prácticas más importantes de los seminarios que celebran gira en torno al conocimiento de uno mismo y al desarrollo de valores personales. MeSumaría desarrolla proyectos que contribuyen a la adquisición de estos valores; un ejemplo es Con los Mayores, programa en el que el alumnado colabora y convive con ancianos y ancianas, viéndose fortalecidas la colaboración, el respeto, o la empatía.

Los principios recogidos en los indicadores seis y siete se tratan conjuntamente; en ambos se refleja la defensa de la convivencia intercultural, disminuyéndose las desigualdades sociales. Estas ideas forman parte de las tres experiencias analizadas. Red Khelidôn defiende que, para lograr una cooperación real y efectiva, los grupos que se forman han de 
ser heterogéneos. Parten de que el aprendizaje de la cooperación en grupos que poseen esta diversidad favorece el desarrollo de la convivencia positiva y el respeto entre los miembros de cada equipo. En Educación en Transición se deduce la heterogeneidad de los agrupamientos, pues no existen requisitos para poder participar en las prácticas que, promovidas desde los seminarios, se realizan, dando así la oportunidad de formar parte de la experiencia a todos los miembros de la sociedad.

Por último, el trabajo de ambos indicadores se torna indiscutible en el caso de MeSumaría, viéndose reflejado en todos sus proyectos, como, por ejemplo, en Desde la Diversidad o en Vidas Cruzadas. En el primero existen agrupaciones heterogéneas formadas por personas de diferente procedencia, edad, sexo, clase socioeconómica o cultura. En Vidas Cruzadas participan grupos formados por presidiarios y adolescentes, quienes comparten sus experiencias y reflexiones utilizando relatos expuestos en un blog.

Las tres experiencias contribuyen al desarrollo del octavo indicador, referido a la educación en el conflicto. Red Khelidôn, mediante el enfoque inclusivo de la educación y de la sociedad, cultiva la cohesión de grupo para ayudar al alumnado en la resolución positiva de conflictos. Educación en Transición incide en el pensamiento crítico o la reflexión que puede derivar de la visión positiva de un conflicto. Partiendo del problema medioambiental, sociopolítico y económico, promueven seminarios en los que nacen reflexiones críticas dirigidas al cambio.

Ejemplificamos el octavo indicador en MeSumaría, haciendo referencia a una situación concreta. Cuando llevaron a cabo un proyecto en Tiétar (Cáceres), se encontraron con que existía una división entre los autóctonos y la población marroquí, lo cual denotaba una concepción racista y cultural negativa; partiendo del conflicto y poniendo en marcha sus principios y actuaciones, lograron que ambos grupos mejorasen su convivencia.

El noveno indicador, relacionado con la educación para la desobediencia y el desarrollo del pensamiento crítico y libre del alumnado, constituye un objetivo a alcanzar por Red Khelidôn, quien lo trabaja de forma parcial. En el momento de analizar la experiencia en base a este principio ha existido cierta dificultad, pues cada docente lleva a cabo la práctica de los proyectos en su aula; esto implica que el hecho de permitir que el alumnado exprese sus propias opiniones depende en gran medida de cada docente. El trabajo cooperativo favorece la opinión en pequeño grupo, pero el trabajo de Red Khelidôn con centros confesionales, difícilmente dará lugar al desarrollo del pensamiento libre y crítico cuando contradiga los ideales de la propia escuela.

En el caso de Educación en Transición y MeSumaría, el indicador se desarrolla totalmente. El primero de ellos defiende la preeminencia de la educación para la desobediencia, promoviendo el desarrollo de un pensamiento crítico y libre a través de sus prácticas; se observa, por ejemplo, en las manifestaciones protagonizadas por estudiantes de diferentes escuelas e institutos con motivo del cambio climático.

A su vez, lo establecido en el indicador comprende uno de los objetivos principales de MeSumaría, pues el modelo crítico y reflexivo, basado en la libertad de expresión, forma parte de su base pedagógica principal. Debido a la diversidad y amplitud de propuestas desarrolladas, resultaría difícil conseguirlo en todas ellas, pero queda latente que intentan alcanzar la creación de un pensamiento crítico y libre, y que en algunas ocasiones lo consiguen. 
Continuamos el análisis expresando que el décimo indicador, en el cual se defiende la igualdad de oportunidades y la lucha contra la injusticia o el racismo, se alcanza en Red Khelidôn y en MeSumaría a través del trabajo en equipo. Además, desde ambas experiencias se asume un modelo de educación inclusiva que acepta a todo el alumnado independientemente de sus peculiaridades. Las propuestas de Educación en Transición están encaminadas al desarrollo de una sociedad más humana, justa e igualitaria. No obstante, están más dirigidas al desarrollo de una armonía con la naturaleza y a la lucha en favor de cuestiones medioambientales, por lo que no se actúa directamente contra otros factores como el racismo, la pobreza o el poder centralizado; por todo ello, el indicador se cumple parcialmente.

La participación de todas las personas que forman la comunidad educativa -indicador 11es otro de los principios fundamentales de la educación democrática. Según los estatutos de Red Khelidôn, existen diferentes órganos de gobierno; entre ellos, la Asamblea General, formada por todos los miembros que componen la Red. Podríamos afirmar que el indicador se cumple, pero existen algunas matizaciones por las que exponemos que se alcanza parcialmente: emplean el voto para tomar decisiones, otorgando el derecho a un voto únicamente si hay cinco docentes de un mismo colegio adheridos, o a cada centro en el caso de que la totalidad de docentes del mismo formen parte de la Red.

Educación en Transición y MeSumaría reflejan lo expresado en el undécimo indicador de forma total. El primero lo consigue en experiencias concretas, en las que se observa que las opiniones de los participantes se tienen en cuenta, sobre todo, a nivel de propuesta. Asimismo, podemos nombrar la experiencia de Jóvenes por el Clima, formada por miembros del alumnado y por docentes, en la cual las opiniones de todos los componentes están al mismo nivel y reciben la misma importancia. En el segundo caso, existe colaboración por parte de todas las personas que conforman MeSumaría. Para ello, emplean el espacio deliberativo democrático por excelencia: la asamblea; en ella realizan propuestas, evalúan y comparten experiencias.

La concepción de los estudiantes como centro y destino de toda actividad educativa indicador 12- se refleja en las tres experiencias. El presente indicador se abarca de forma parcial en Educación en Transición y MeSumaría, y de forma total en Red Khelidôn. Esta última está destinada a lograr la mejora de las prácticas educativas mediante el trabajo cooperativo, formando ciudadanos libres, críticos, activos, comprometidos con la sociedad y poseedores de unas competencias y unos valores esenciales para contribuir a la generación de una sociedad justa, equitativa e inclusiva. Todo el proyecto está destinado a desarrollar las potencialidades de los educandos.

El indicador no se refleja completamente en Educación en Transición, pues su objetivo último no es el alumnado en concreto, sino contribuir al avance en la educación para crear un mundo más igualitario y justo. Cierto es que, a través del cambio o la transición de la educación, se mejorará su calidad y el desarrollo de las potencialidades del alumnado, pero este no es su principal objetivo.

En el caso de MeSumaría, el educando comprende no solo a los niños y las niñas, sino a otros colectivos de la sociedad (docentes, familias, ancianos, etc.). Si identificamos a estos agentes como "alumnado", diríamos que el indicador se cumple; no obstante, si consideramos que "los discentes" son los estudiantes en edad escolar, deberíamos matizar, 
debido a que no en todos los proyectos son los máximos protagonistas. Por ello, consideramos que este indicador se satisface de manera parcial.

$\mathrm{El}$ indicador relacionado con el desarrollo integral de los educandos -indicador 13- se alcanza en las tres experiencias. En Red Khelidôn el trabajo cooperativo incide en la formación moral (solidaridad, respeto, empatía, igualdad, etc.), en la formación cognoscitiva (trabajo en equipo como contenido) y en la formación profesional (la cohesión de grupo y aprender a trabajar en equipo). En Educación en Transición también encontramos prácticas de carácter moral (educación medioambiental, armonía con la naturaleza, habilidad de alteridad, capacidad de amar, etc.), cognoscitivo (el cambio climático y el capitalismo como objeto de estudio, los huertos, la moneda, etc.) y profesional (proactividad, gestión de huertos, debates, intercambio de monedas complementarias, taller de bicicletas, etc.).

En MeSumaría extraemos ejemplos de cada tipo de formación: 1) Formación moral: trabajo de la diversidad (Escuela MeSumaría), la educación emocional (actividades para gestionar, conocer y expresar emociones: ImaginArte), la formación holística y la conciencia social. 2) Formación cognoscitiva: se trabajan contenidos académicos mediante técnicas diversas. Por ejemplo, en Teatro en la Escuela, se trabajan contenidos de diferentes áreas del currículo, empleando el teatro como recurso. En Vidas Cruzadas e ImaginArte se crean composiciones escritas. Asimismo, se abarca la sociedad de la información utilizando recursos tecnológicos y TIC; un ejemplo lo encontramos en Navegamos Juntos, donde adolescentes muestran a las personas ancianas cómo navegar por Internet. 3) Formación profesional: incluye aspectos como aprender a trabajar en equipo, potenciar la creatividad o aprender a utilizar de forma adecuada las TIC.

$\mathrm{El}$ indicador destinado al conocimiento personal, social y familiar de los destinatarios de las propuestas -número 14-, se alcanza totalmente en Red Khelidôn y MeSumaría. En el primero, cuando se organizan los Equipos Base, el profesorado tiene en cuenta todas las variables de diversidad, las circunstancias personales, sociales y familiares de cada estudiante. Del mismo modo, MeSumaría afirma conocer las circunstancias concretas de los individuos con los que trabaja en cada proyecto.

En Educación en Transición se ve satisfecho de forma parcial, puesto que colaboran con diferentes centros y grupos de estudiantes, lo que dificulta la posibilidad de tener en cuenta las circunstancias individuales, personales, sociales y familiares de cada participante, pero sus propuestas son flexibles, pudiendo ser alteradas y modificadas según las necesidades de cada momento.

El indicador número 15, el cual abarca la conexión con el entorno, se satisface en Red Khelidôn y en Educación en Transición. En Red Khelidôn las interacciones con otras personas o centros son constantes; uno de sus objetivos consiste en lograr el intercambio de experiencias y recursos entre participantes. Actualmente hay Equipos Coordinadores de Zona en cinco comunidades autónomas, favoreciéndose la relación entre profesionales. En MeSumaría existe una relación de los participantes con el entorno social y cultural, pero en ninguna de las bases de sus proyectos hace referencia al medio natural. Debido a esto, el indicador se cumple parcialmente. En Educación en Transición el indicador se consigue, pues uno de sus objetivos es el establecimiento de una relación real, positiva y permanente con la naturaleza mediante diferentes actividades (huertos escolares, abrazar árboles, escucha del medio, etc.). 
Finalizamos el análisis afirmando que el último indicador, el cual abarca el carácter autónomo de las prácticas educativas, aparece en todas las experiencias. Red Khelidôn posee cierto autogobierno; está dirigida por "Educación inclusiva, cooperación entre alumnos y colaboración entre profesores" del Centro de Innovación y Formación en Educación de la Universidad de Vic y tiene su propio equipo de gobierno. Cada zona puede organizarse en función de las necesidades de los centros de cada comunidad y existe la posibilidad de modificar los documentos vigentes.

Los documentos sobre los que se sustenta Educación en Transición también son abiertos y flexibles; se admiten modificaciones que contribuyan a la consecución de sus objetivos. Además, la experiencia es autónoma en sí misma y está vinculada en red con otras asociaciones, donde todos tienen el mismo grado de decisión y nivel de participación. MeSumaría es una asociación que trata de autogobernarse y colaborar con otras entidades y movimientos como con MRP de Extremadura o Escuelas en Red, las cuales se nutren y refuerzan sus proyectos. Esto, y su trabajo mediante asambleas, nos conduce a afirmar que sus documentos y programas son flexibles, pudiendo ser modificados. En la figura 1 se representa gráficamente el grado de democratización de las experiencias educativas analizadas.

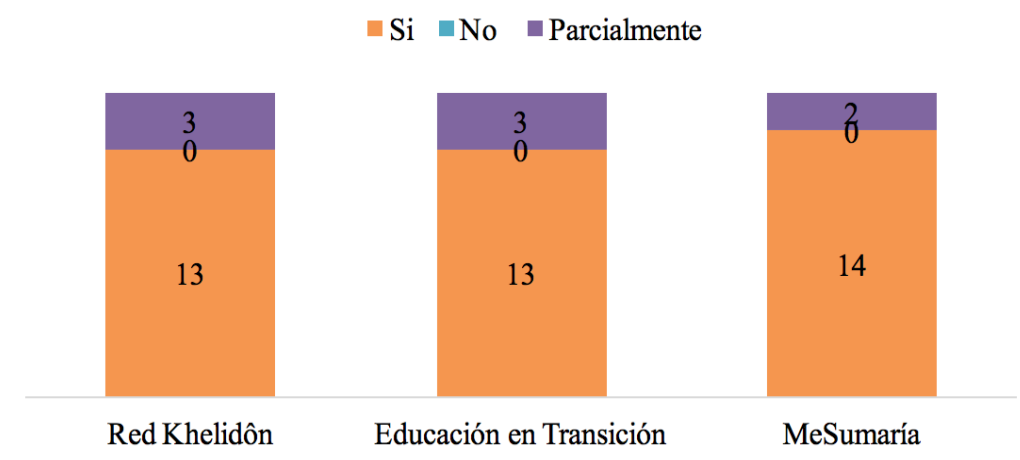

Figura 1. Grado de democratización de las experiencias educativas

Fuente: Elaboración propia.

\section{Discusión y conclusiones}

La búsqueda en las principales bases de datos científicas ha evidenciado la inexistencia de investigaciones directamente orientadas a valorar el grado de democratización de experiencias educativas. No obstante, los rasgos principales de la educación democrática están presentes en la práctica de las experiencias analizadas, aunque con desigual densidad (cuadro 1). Los elementos de la teoría de la educación democrática, recogidos de la literatura científica, son, por tanto, trasladables a la práctica, tal y como se ha mostrado en el estudio.

En líneas generales, creemos conveniente elaborar una valoración positiva, visto que las tres experiencias muestran signos de esperanza, de ilusión por llegar a construir prácticas y escuelas educativas verdaderamente democráticas. En todos los casos, aspectos relevantes para la constitución de una escuela democrática se ven satisfechos, como son el favorecimiento de relaciones sociales, la práctica de actividades colaborativas, el logro de un clima adecuado, la creación de agrupaciones heterogéneas o el respeto intercultural. 
Cuadro 1. Resultados del análisis: ¿Aparecen reflejados los indicadores en cada experiencia?

\begin{tabular}{lllllllllllllllll}
\hline & $\mathbf{1}$ & $\mathbf{2}$ & $\mathbf{3}$ & $\mathbf{4}$ & $\mathbf{5}$ & $\mathbf{6}$ & $\mathbf{7}$ & $\mathbf{8}$ & $\mathbf{9}$ & $\mathbf{1 0}$ & $\mathbf{1 1}$ & $\mathbf{1 2}$ & $\mathbf{1 3}$ & $\mathbf{1 4}$ & $\mathbf{1 5}$ & $\mathbf{1 6}$ \\
\hline Red Khelidôn & $\mathrm{P}$ & $\mathrm{Si}$ & $\mathrm{Si}$ & $\mathrm{Si}$ & $\mathrm{Si}$ & $\mathrm{Si}$ & $\mathrm{Si}$ & $\mathrm{Si}$ & $\mathrm{P}$ & $\mathrm{Si}$ & $\mathrm{P}$ & $\mathrm{Si}$ & $\mathrm{Si}$ & $\mathrm{Si}$ & $\mathrm{Si}$ & $\mathrm{Si}$ \\
\hline $\begin{array}{c}\text { Educación en } \\
\text { Transición }\end{array}$ & $\mathrm{Si}$ & $\mathrm{Si}$ & $\mathrm{Si}$ & $\mathrm{Si}$ & $\mathrm{Si}$ & $\mathrm{Si}$ & $\mathrm{Si}$ & $\mathrm{Si}$ & $\mathrm{Si}$ & $\mathrm{P}$ & $\mathrm{Si}$ & $\mathrm{P}$ & $\mathrm{Si}$ & $\mathrm{P}$ & $\mathrm{Si}$ & $\mathrm{Si}$ \\
\hline MeSumaría & $\mathrm{Si}$ & $\mathrm{Si}$ & $\mathrm{Si}$ & $\mathrm{Si}$ & $\mathrm{Si}$ & $\mathrm{Si}$ & $\mathrm{Si}$ & $\mathrm{Si}$ & $\mathrm{Si}$ & $\mathrm{Si}$ & $\mathrm{Si}$ & $\mathrm{P}$ & $\mathrm{Si}$ & $\mathrm{Si}$ & $\mathrm{P}$ & $\mathrm{Si}$ \\
\hline
\end{tabular}

Nota: "P": el indicador se satisface parcialmente. "Si": el indicador se satisface totalmente. "No": el indicador no se refleja en la experiencia.

Fuente: Elaboración propia.

No obstante, existen indicadores que se reflejan en alguna de las experiencias de forma parcial, tal vez los más complejos de abordar, como el relacionado con el pensamiento crítico y libre del alumnado. Esto puede indicarnos el temor existente ante la rebelión o la libre expresión de ideas que contradigan las arraigadas en la concepción hegemónica de la sociedad, generalmente ideas arcaicas, autoritarias, propias de un sistema educativo caduco y obsoleto. Torrego (2013) coincide con este pensamiento, exponiendo que:

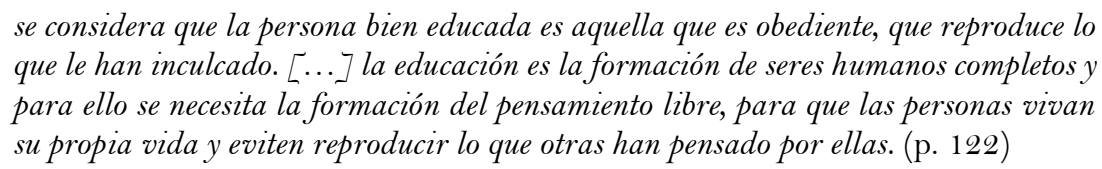

Esta idea también la defiende Yus (2002), afirmando que debe superarse la tendencia de dejar que otras personas tomen las decisiones por nosotros, "bien por comodidad o bien porque así siempre ha sido" (p. 15). Llamas y Gorbe (2014) comparten esta percepción y añaden que el desarrollo del pensamiento crítico y libre constituye un problema en la educación en España a causa de "la compartimentación de la enseñanza, la falta de globalización y relación con la vida del aprendizaje escolar, que necesita una solución urgente en la línea del cambio organizativo y metodológico” (p. 567).

Por otra parte, nos aventuramos a afirmar que el desarrollo de este indicador será complejo en una escuela de carácter confesional; una escuela libre, democrática y justa no puede estar marcada por ninguna ideología. Por todo esto, y como vienen defendiendo los MRP, para conseguir escuelas realmente democráticas, debemos potenciar el desarrollo de centros educativos públicos, laicos, solidarios, científicos e inclusivos, accesibles para todos los ciudadanos. Con todo ello coincide Rogero (2010) al hablarnos sobre los principios que configuran las ideas educativas de los MRP.

Además, hemos tenido la evidencia de que el alumnado no participa activamente en la planificación de las actividades a pesar de ser el protagonista, centro y destino de las mismas; coincidiendo con Parareda, Serra y Tort (2015), "la participación [ ...] también significa incluir al alumnado en procesos democráticos o de toma de decisiones en la escuela en los que tengan voz y representación” (p. 16), haciéndoles partícipes y responsables de la propia institución.

En conclusión, aunque aún deben desarrollarse numerosos aspectos en algunas de las experiencias, es evidente que se está avanzando adecuadamente hacia lo que entenderíamos como práctica democrática, pues los resultados han sido realmente positivos. Las tres experiencias educativas pueden ser consideradas democráticas, lo cual denota que el modelo de educación que persiguen los MRP es posible, y nos permite 
transmitir a la sociedad la importancia que tienen las actuaciones y las iniciativas que estos desarrollan para alcanzar una mejora real en la educación.

\section{Referencias}

Apple, M. W. y Beane, J. A. (1997). Escuelas democráticas. Madrid: Morata.

Belavi, G. y Murillo, F. J. (2016). Educación, democracia y justicia social. Revista Internacional de Educación para la Justicia Social, 5(1), 13-34. https://doi.org/10.15366/riejs2016.5.1

Biesta, G. (2016). Democracia, ciudadanía y educación: De la socialización a la subjetivación. Foro de Educación, 14(20), 21-34. https://doi.org/10.14516/fde.2016.014.020.003

Biesta, G. y Lawy, R. (2006). From teaching citizenship to learning democracy: Overcoming individualism in research, policy and practice. Cambridge Journal of Education, 36(1), 63-79. https://doi.org/10.1080/03057640500490981

Bolívar, A. (2016). Educar democráticamente para una ciudadanía activa. Revista Internacional de Educación para la Justicia Social, 5(1), 69-87. https://doi.org/10.15366/riejs2016.5.1

Carr, P. R., Pluim, G. y Thésée, G. (2017). The dimensions of, and connections between, multicultural social justice education and education for democracy: What are the roles and perspectives of future educators? Citizenship Education Research Journal, 6(1), 3-23.

Checkoway, B. (2013). Education for democracy by young people in community-based $\begin{array}{llll}\text { organizations. } & \text { South } & \text { S } & \text { Society, }\end{array}$ https://doi.org/10.1177/0044118X11419535

Domínguez, J. (s.f.). La escuela democrática. Recuperado de https://goo.gl/1Z1fqe

Domínguez, J. (2006). Un currículo democrático. Democratizar los currículos. Recuperado de https://goo.gl/fd3oGO

Domínguez, J. (2008). Centros democráticos. Los centros educativos democráticos como ecosistemas necesarios para los currículos democráticos. Recuperado de https://goo.gl/rofGW9

Edelstein, W. (2011). Education for democracy: reasons and strategies. European Journal of Education, 46(1), 127-137. https://doi.org/10.1111/j.1465-3435.2010.01463.x

Feito, R. (2009). Escuelas democráticas. Revista de la Asociación de Sociología de la Educación, 2(1), $17-33$.

Feito, R. y López, J. I. (2008). Construyendo escuelas democráticas. Barcelona: Hipatia.

Guba, E. G. (1983). Criterios de credibilidad en la investigación naturalista. En J. Gimeno y A. Pérez (Coords.), La enseñanza: Su teoría y su práctica (pp. 148-165). Madrid: Akal.

Hansen, D. T. y James, C. (2016). The importance of cultivating democratic habits in schools: Enduring lessons from democracy and education. Journal of Curriculum Studies, 48(1), 94112. https://doi.org/10.1080/00220272.2015.1051120

Llamas, E. y Gorbe, P. (2014). Pensamiento crítico: Una propuesta educativa para educación primaria. En J. J. Maquilón, A. Escarbajal y R. Nortes (Eds.), Vivencias innovadoras en las aulas de primaria (pp. 557-570). Murcia: Universidad de Murcia.

Martínez, J. (1993). Los MRPs o el compromiso en la escuela. Cuadernos de Pedagogía, 220, 104109.

Martínez, J. (2002). ¿Qué son los MRP? Cuadernos de Pedagogía, 311, 85-89. 
Mejía, J. (2000). El muestreo en la investigación cualitativa. Investigaciones Sociales, 4(5), 165-180.

Navarro, R. (1989). El franquismo, la escuela y el maestro (1936-1975). Historia de la educación: Revista Interuniversitaria, 8, 167-180.

Parareda, A., Serra, C. y Tort, A. (2015). La democracia y la participación en los centros escolares. Aula de Innovación Educativa, 243, 12-16.

Pericacho, F. J. (2014). Pasado y presente de la renovación pedagógica en España (de finales del siglo XIX a nuestros días). Un recorrido a través de escuelas emblemáticas. Revista Complutense de Educación, 25(1), 47-67.

Pericacho, F. J. (2015). Actualidad de la renovación pedagógica en la Comunidad de Madrid: Un estudio a través de escuelas emblemáticas. Evolución y experiencias actuales ante los retos socioeducativos de la sociedad del siglo XXI. Tesis doctoral. Universidad Complutense, Madrid.

Piñuel, J. L. (2002). Epistemología, metodología y técnicas del análisis de contenido. Estudios de Sociolingüística, 3(1), 1-42.

Puig, J. M. (2000). ¿Cómo hacer escuelas democráticas? Educaçao e Pesquisa, 26(2), 55-69. https://doi.org/10.1590/S1517-97022000000200005

Rogero, J. (2010). Movimientos de renovación pedagógica y profesionalización docente. Revista Latinoamericana de Educación Inclusiva, 4(1), 141-166.

Serbia, J. M. (2007). Diseño, muestreo y análisis en la investigación cualitativa. HologramáticaFacultad de Ciencias Sociales, 3(7), 123-146.

Torrego, L. M. (1999). Canción de autor y educación popular (1960-1980). Madrid: Ediciones de la Torre.

Torrego, L. (2013). Defendiendo lo colectivo: Combatir el conformismo, promover la educación pública. Revista Interuniversitaria de Formación del Profesorado, 78(27,3), 115-123.

Torrego, L. (2014). ¿Investigación difusa o emancipatoria? Participación e inclusión en investigación educativa. Magis: Revista Internacional de Investigación en Educación, 7(14), 113 124. https://doi.org/10.11144/Javeriana.M7-14.IDEP

Yus, R. (2002). Educación integral y educación democrática. Barbecho. Revista de Reflexión Socioeducativa, 1, 12-15.

\section{Breve CV de los autores}

\section{Rosa Ortiz de Santos}

Graduada en Educación Primaria y titulada en el Máster de investigación en ciencias sociales. Educación, comunicación audiovisual, economía y empresa por la Universidad de Valladolid. Actualmente doctoranda en el Programa de Doctorado en Investigación Transdisciplinar en Educación y contratada predoctoral en el Departamento de Pedagogía de la Facultad de Educación de Segovia (Universidad de Valladolid, España). Líneas de investigación: renovación pedagógica, educación democrática e historia de la educación. ORCID ID: 0000-0003-2408-1645. Email: rosa.ortiz@uva.es

\section{Luis Torrego}

Doctor en Filosofía y Ciencias de la Educación por la UNED. Profesor Titular de Universidad en el Departamento de Pedagogía de la Universidad de Valladolid. Coordinador y Presidente de la Comisión Académica del Programa de Doctorado en 
Investigación Transdisciplinar en Educación de la Universidad de Valladolid. Autor o coautor de 77 artículos en revistas científicas indexadas (fuente: Google Scholar), coautor y coordinador del libro "Educación inclusiva, equidad y derecho a la diferencia" (2013, Editorial Wolters Kluwer) y director de 25 tesis doctorales defendidas. Sus líneas de investigación son: Educación inclusiva, Renovación pedagógica y Educación para la paz y la igualdad. ORCID ID: 0000-0002-2907-1158. Email: 1torrego@pdg.uva.es

\section{Noelia Santamaría-Cárdaba}

Graduada en Educación Primaria por la especialidad de Entorno, Naturaleza y Sociedad y titulada en el Máster de investigación en ciencias sociales. Educación, comunicación audiovisual, economía y empresa por la Universidad de Valladolid. Actualmente doctoranda en el Programa de Doctorado en Investigación Transdisciplinar en Educación y contratada predoctoral en el Departamento de Sociología de la Facultad de Ciencias Sociales, Jurídicas y de la Comunicación (Universidad de Valladolid, España). Sus líneas de investigación son: Educación para el desarrollo, Renovación pedagógica y Educación para la paz y la igualdad. ORCID ID: 0000-0001-6864-9330. Email: noelia.santamaria.cardaba@uva.es 